\title{
Mortalidad y proyecciones por causas de muerte en el Estado de México, 1980-2050
}

\section{Mortality and projections by causes of death in the State of Mexico, 1980-2050}

\author{
Yuliana Gabriela Román-Sánchez \\ Hugo Montes de Oca-Vargas \\ José Antonio Soberón-Mora \\ Centro de Investigación y Estudios Avanzados de la Población \\ de la Universidad Autónoma del Estado de México, México
}

\section{Resumen}

El presente artículo tiene como objetivo analizar la mortalidad por causas de la población mexiquense de 1980 a 2015 y estimar proyecciones de la mortalidad según siete principales causas de muerte para el año 2050. Para realizar lo anterior, se utilizaron cuatro fuentes de datos: Estadísticas vitales, Censos de Población y Vivienda, Conteos de Población y Vivienda y la Encuesta Intercensal (2015). Asimismo, se estimaron tasas específicas de mortalidad estandarizadas por edad y sexo, en tanto que las proyecciones de mortalidad por causa de muerte se calcularon utilizando el método de estimación Gamma. Los resultados sugieren que las principales causas de muerte para el 2050 serán aquellas que hasta ahora son enfermedades no transmisibles, o también llamadas crónico degenerativas. Lo cual puede traer como consecuencias el incremento de gastos económicos en salud, atención médica, el aumento en las horas para el cuidado de adultos mayores. Todo esto podría producir cambios en los roles y cargas de trabajo de la sociedad.

Palabras clave: Envejecimiento, mortalidad por causas, proyecciones de mortalidad, Estado de México.

\section{Abstract}

The main objective of this article is to analyze the mortality by causes the population of State of Mexico from 1980 to 2015, and to estimate projections of mortality according to seven main causes of death for the year 2050. To do the above, four data sources were used: Vital statistics, Population and Housing Censuses, Population and Housing Counts and the Intercensal Survey (2015). Likewise, specific mortality rates standardized by age and sex were estimated, while mortality projections by cause of death were calculated using the Gamma estimation method. The results suggested that the main causes of death by 2050 will be those that until now are non-communicable diseases, or also called chronic degenerative diseases. Which can bring as consequences the increase of economic expenses in health, medical attention, the increase in the hours for the care of older adults. All this could lead to changes in the roles and workloads of society.

Key words: Mortality by causes, mortality projections, state of Mexico 


\section{INTRODUCCIÓN}

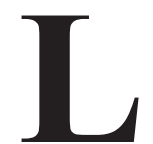

a mortalidad es uno de los componentes demográficos que se utiliza para analizar el crecimiento de una población en un tiempo determinado. Asimismo, permite visualizar hechos pasados, su impacto en la estructura por edad y sexo, y permite discernir lo que podría ocurrir en el futuro en la pirámide poblacional.

El presente artículo tiene como objetivo analizar la mortalidad por causas de la población mexiquense de 1980 a 2015, así como estimar las proyecciones de los principales grupos de causas de muerte para el año 2050. Este tema es relevante por diversas razones: i) permite analizar las causas de muerte en la población y a su vez eso posibilita definir propuestas de políticas públicas con énfasis en mejorar la atención médica y salud; ii) hace posible conocer el costo social y económico que conllevan el predominio de causas de muerte por enfermedades crónica degenerativas en población en proceso de envejecimiento; y finalmente, iii) hace posible estimar proyecciones de mortalidad por causa de muerte para visualizar probables escenarios futuros de necesidades y demandas en servicios de atención médica y cuidados.

De acuerdo con las proyecciones de mortalidad del Consejo Nacional de Población (CONAPO, 2020), las defunciones para el año 2020 de la población mexiquense ascendieron a 96,854 , de las cuales 54.08 por ciento corresponden a hombres y el resto 45.92 por ciento a mujeres. Por grupo de edad, las defunciones de los menores de 15 años representaron 5.22 por ciento del total de muertes, 4.1 por ciento de los jóvenes (15 a 29 años), 26.28 por ciento de los adultos ( 30 a 59 años) y 64.2 por ciento corresponde a adultos mayores (60 años o más), lo anterior como consecuencia de una esperanza de vida más prolongada que ha dado inicio al denominado proceso de envejecimiento demográfico.

Bajo este contexto, el presente artículo tiene como preguntas de investigación: ¿Qué causas de muerte tuvieron mayor impacto en la mortalidad de la población del Estado de México durante el periodo 1980-2015? ¿Cómo ha sido el comportamiento de la mortalidad según diferentes grupos de causas de muerte durante ese periodo? ¿Cuál será un posible escenario de la mortalidad por causas para el año 2050 para la población mexiquense?

La investigación tiene un enfoque cuantitativo y de largo plazo. Se utilizaron cuatro fuentes de datos: Estadísticas vitales (1979, 1980, 1981, 1989, 1990, 1991, 1999, 2000, 2001, 2009, 2010, 2011), Censos de Población y Vivienda (1980, 1990, 2000 y 2010), Conteos de Población y Vivienda 
(1995, 2005) y la Encuesta Intercensal (2015). Se estimaron tasas específicas estandarizadas de mortalidad por edad y sexo. Estas herramientas son esenciales para analizar el patrón y comportamiento de la mortalidad.

Los siete grupos de causas de muerte que se analizaron y proyectaron fueron: i) ciertas enfermedades infecciosas y parasitarias; ii) tumores o neoplasias; iii) enfermedades endócrinas; iv) enfermedades del sistema circulatorio; v) enfermedades del sistema respiratorio; vi) enfermedades del sistema digestivo; vii) causas externas de mortalidad y morbilidad. Esta clasificación la utiliza el Instituto Nacional de Estadística y Geografía (INEGI) y forma parte de la Clasificación Internacional de Enfermedades versión 10 (CIE, 10).

Parte de la justificación de usar estos grupos de causas de muerte es que para el año 2019 , dichas causas provocaron un total de 71,762 defunciones entre la población mexiquense, de las cuales 56.02 por ciento se presentaron en varones y 43.97 por ciento en mujeres. Estas muertes representaron 89.24 por ciento de las defunciones total en la entidad. Otro dato interesante muestra que una de cada dos muertes ocurridas en el Estado de México fue por una causa relacionada con el sistema endócrino o sistema respiratorio (INEGI, 2020).

El artículo se compone de cuatro apartados y la sección de conclusiones. El primero expone los fundamentos teóricos de la teoría de la transición epidemiológica, aspectos indispensables para entender el contexto actual. El segundo se centra en exponer el comportamiento de siete causas de muerte de la población mexiquense para el periodo de tres décadas y media. El tercer apartado muestra la metodología para estimar las proyecciones de la mortalidad por causas para los años 2020, 2030, 2040 y 2050 en la entidad mexiquense. La cuarta sección expone y analiza las proyecciones de la mortalidad según causa para el periodo propuesto e incluye la discusión de los principales resultados.

\section{TEORÍA DE LA TRANSICIÓN EPIDEMIOLÓGICA}

La idea central de la teoría de la transición epidemiológica se concentra en el estudio de los patrones de las causas de muerte en la población, y con ello es posible analizar el proceso de los cambios en la mortalidad. Un punto esencial es que la proporción de las muertes y enfermedades se desplaza de los grupos de edades jóvenes a los de edades avanzadas, y en este contexto el aumento o disminución en términos relativos y absolutos de cada segmento por edad de la población en un país pueden repercutir de 
cierta forma en los patrones de mortalidad (Organización Panamericana de la Salud, 2001).

Existen diversas definiciones sobre la teoría de la transición epidemiológica: según Vera (1999) esta teoría se postula como el cambio de un patrón de causas de muerte dominado por enfermedades infecciosas, con muy alta mortalidad especialmente en las edades jóvenes, a un patrón caracterizado por enfermedades degenerativas y afecciones producidas por la acción del hombre, como las formas más importantes de morbilidad y causa de muerte.

Por su parte Frenk, Frejka y Bobadilla (1991) la definen de manera más amplia; ellos involucran los cambios de frecuencia, magnitud y distribución de las condiciones de salud, expresadas en términos de muerte, enfermedad e invalidez. Además, implica un cambio en una dirección predominante: de las enfermedades infecciosas asociadas con carencias primarias a las enfermedades crónicas y degenerativas, lesiones y padecimientos mentales, todos estos relacionados con padecimientos genéticos y carencias secundarias.

Paralelamente, Cabrera (2010) afirma que, Omran — conocido como el padre de la Transición Epidemiológica - acota el término de transición epidemiológica, para sugerir que la transición era un proceso continuo en el cual los patrones de salud y enfermedad de una sociedad se van transformando, en respuesta a cambios más amplios de carácter demográfico, socioeconómico, tecnológico, político, cultural y biológico. En un periodo caracterizado en un principio de enfermedades infectocontagiosas y finalmente hacia los padecimientos no transmisibles.

Esta teórica también ha sido objeto de ciertas críticas. Al respecto Riley y Alter (1990) plantean que lo que Omran observó con los datos históricos era, más que una transición en los patrones de enfermedad, una transición en la edad a la que se producía la muerte, como resultado de este cambio una proporción mayor de cada cohorte ha ido sobreviviendo a edades en que las enfermedades no transmisibles, crónicas y degenerativas reemplazan a las transmisibles como principal causa de muerte (Bernabeu y Robles, 2000).

Asimismo, otra crítica recae en que estos planteamientos teóricos fueron formulados en 1971 cuando la epidemia del Síndrome de Inmunodeficiencia Adquirida (SIDA) y otras enfermedades infecciosas emergentes como la tuberculosis y el ébola aún no se habían detectado. Dichas enfermedades, de tipo infeccioso, surgieron cuando se creía haber logrado el control sobre las enfermedades transmisibles (Vera, 1999). Lo mismo 
ocurre con el virus SARs-CoV-2 que ha dejado en poco tiempo millones de infectados y defunciones por todo el mundo.

En ese mismo sentido, la Organización Panamericana de la Salud (OPS, 2001) afirma que la propuesta original del modelo de transición epidemiológica asumía una evolución lineal de los cambios epidemiológicos; la historia reciente ha demostrado la reversibilidad de éstos, su naturaleza no lineal y la coexistencia de enfermedades no transmisibles y transmisibles.

En la actualidad, una tendencia que se está presentando es el resurgimiento de algunas enfermedades infecciosas como formas de morbilidad y en la población. Con ello se pone en evidencia lo que postula la transición epidemiológica, ya que en algunos países no se han logrado de erradicar por completo las enfermedades infecciosas cuando las enfermedades crónicas degenerativas se han posicionado como principales causas de morbilidad, lo que demuestra que existe un perfil epidemiológico mixto de ambos tipos de enfermedades, las cuales influyen en los patrones de morbilidad que presente una sociedad específica, cuyos efectos repercuten principalmente en los sistemas de salud.

El 2020 ha sido denominado como el año de la pandemia por el virus SARs-CoV-2, mejor conocido como coronavirus o Covid-19, éste ha afectado a casi todos los países del mundo. Hasta el 6 de diciembre de 2020 el panorama mundial aún se observa crítico. Las cifras muestran que 66'137,304 personas a nivel mundial han sido contagiadas por Covid-19. El número de fallecimientos en el mundo es de 1'522,940 personas, Estados Unidos de América es el país con mayor número de víctimas hasta este momento con 279,253 personas fallecidas, seguido de Brasil, India y México con 175,964, 139,700 y 109,456 defunciones, respectivamente. No obstante, estas cifras se actualizan diariamente y pueden entrar y salir países con mayores defunciones.

En México el inicio de la pandemia comenzó a finales del mes de marzo de 2020, cuando el Gobierno Federal hizo el llamado de quedarse en casa, suspendiendo a nivel federal todas las actividades académicas y económicas no esenciales; lo anterior después de defunciones detectadas por el virus SARs-CoV-2.

El perfil epidemiológico es la expresión de la carga de enfermedad (estado de salud) que sufre la población, cuya descripción requiere de la identificación de las características que la definen. Entre estas características están la mortalidad, la morbilidad y la calidad de vida (Whittembury, 2007). 
Para la Organización Mundial de la Salud (OMS, 2003) existe una transición epidemiológica mundial evidenciada por el aumento de muertes debido a las nuevas y crecientes enfermedades no transmisibles en comparación con las enfermedades transmisibles que predominaron durante buena parte de nuestra historia. Aunque para ciertas edades en determinados países las enfermedades transmisibles son la primera causa de defunción; por ello, estos países enfrentan una doble carga de morbilidad dado que a la vez de luchar contra las enfermedades infecciosas y contagiosas deben hacerlo contra las no contagiosas (Sime, 2011).

Para América Latina y México se identifica un perfil de salud latinoamericano con el término "modelo polarizado prolongado" caracterizado por: i) sobreposición de etapas (alta incidencia tanto de enfermedades transmisibles como de enfermedades no transmisibles); ii) contratransición (ruptura del principio unidireccional transicional); iii) transición prolongada (situación de estanque epidemiológico, y iv) polarización epidemiológica (heterogeneidad entre grupos sociales y área geográfica interna al mismo país) (Frenk, Frejka y Bobadilla, 1991). En suma, esta perspectiva teórica se considera relevante debido a que se analizan los cambios en las causas de muerte durante tres décadas y media y lo esperado hasta el año 2050.

\section{Nivel de mortalidad en el Estado de México,1980-2015}

El Estado de México es una entidad federativa que se caracteriza por tener una alta participación en la actividad económica del país, debido a su contigüidad con la Ciudad de México, lo anterior hace que dicho Estado tenga un fuerte dinamismo en su economía que incide en la atracción de población, situación que la convierte en una entidad receptora de personas en edad de trabajar (Rózga, 1994). No obstante, esta situación ha cambiado, actualmente es considerada una entidad expulsora de población, sobre todo hacia las ciudades periféricas y en menor medida hacia el extranjero.

Como evidencia de lo anterior, los datos censales muestran que la tasa de crecimiento poblacional más alta se registró entre 1960-1970 fue de 7.3 por ciento, con un tiempo de duplicación de diez años. En 1970 la entidad ocupaba el segundo lugar en número de población, después del Distrito Federal — hoy Ciudad de México—, situación que se mantuvo hasta 1980. Desde 1990 y hasta la fecha el Estado de México es la entidad más poblada del país. La cifra más reciente muestra una tasa de crecimiento de 1.8 por ciento para 2000-2010 con 39 años de duplicación (Pimienta, Vera, Tapia y Orozco, 2015), mientras que para el año 2020 se registró una tasa de crecimiento total de 1.28 por ciento (CONAPO, 2019). 
Si bien las tasas de crecimiento han descendido, la población ha aumentado en términos absolutos, lo que ha provocado que a lo largo del tiempo la estructura por edad se haya trasformado (Figuras 1-4), como consecuencia de la reducción de la fecundidad y el aumento de la esperanza de vida con una cúspide más amplia.

La cúspide de la pirámide poblacional representa el aumento de personas de 60 años o más, lo que se ha denominado envejecimiento demográfico. En la actualidad, éste representa un tema de relevancia mundial que ha modificado la estructura por edad de la población, con implicaciones económicas, financieras, actuariales, epidemiológicas y políticas. Las cuales producen problemas como deficiencias en los sistemas de pensiones, finanzas públicas, pobreza, marginación y vulnerabilidad (Santos, 1996; Villa y Rivadeneira, 2000; Agar, 2001; Romo, 2008; Canales, 2015).

El fenómeno del envejecimiento demográfico es resultado, por un lado, de un proceso de largo plazo del descenso de la natalidad y la mortalidad que modificó la estructura por edad y, por otro, al mejoramiento en la calidad de vida en los años de sobrevivencia (Agar, 2001; Paz, 2010), pero condicionado a los determinantes sociales y económicos de cada país (Ham, 1999).

El Estado de México al ser la entidad más poblada del país, con 18.07 millones al año 2020, concentra a 1.91 millones de adultos mayores, aunque eso no implica que sea la más envejecida. En la entidad se observa como los adultos mayores han tenido un aumento paulatino, pero constante desde la década de 1980 hasta el año 2020; así mismo se prevé que aumente hasta llegar a 2.97 millones en el 2030, situación que modificará la estructura por edad, tal como se observa en las figuras 1-4 (CONAPO, 2019).

Respecto al nivel de mortalidad en la entidad mexiquense en el periodo de 1980 a 2015, la Tabla 1 muestra el comportamiento de los siete grupos de causas de muerte. Al respecto, es posible apreciar para la población total un declive importante de las muertes a través del tiempo para casi todas las causas analizadas, a excepción de las muertes por enfermedades endócrinas, nutricionales y metabólicas, que han presentado incrementos durante el periodo de tres décadas y media, y para las defunciones a causa de tumores que se han mantenido con estabilidad. 


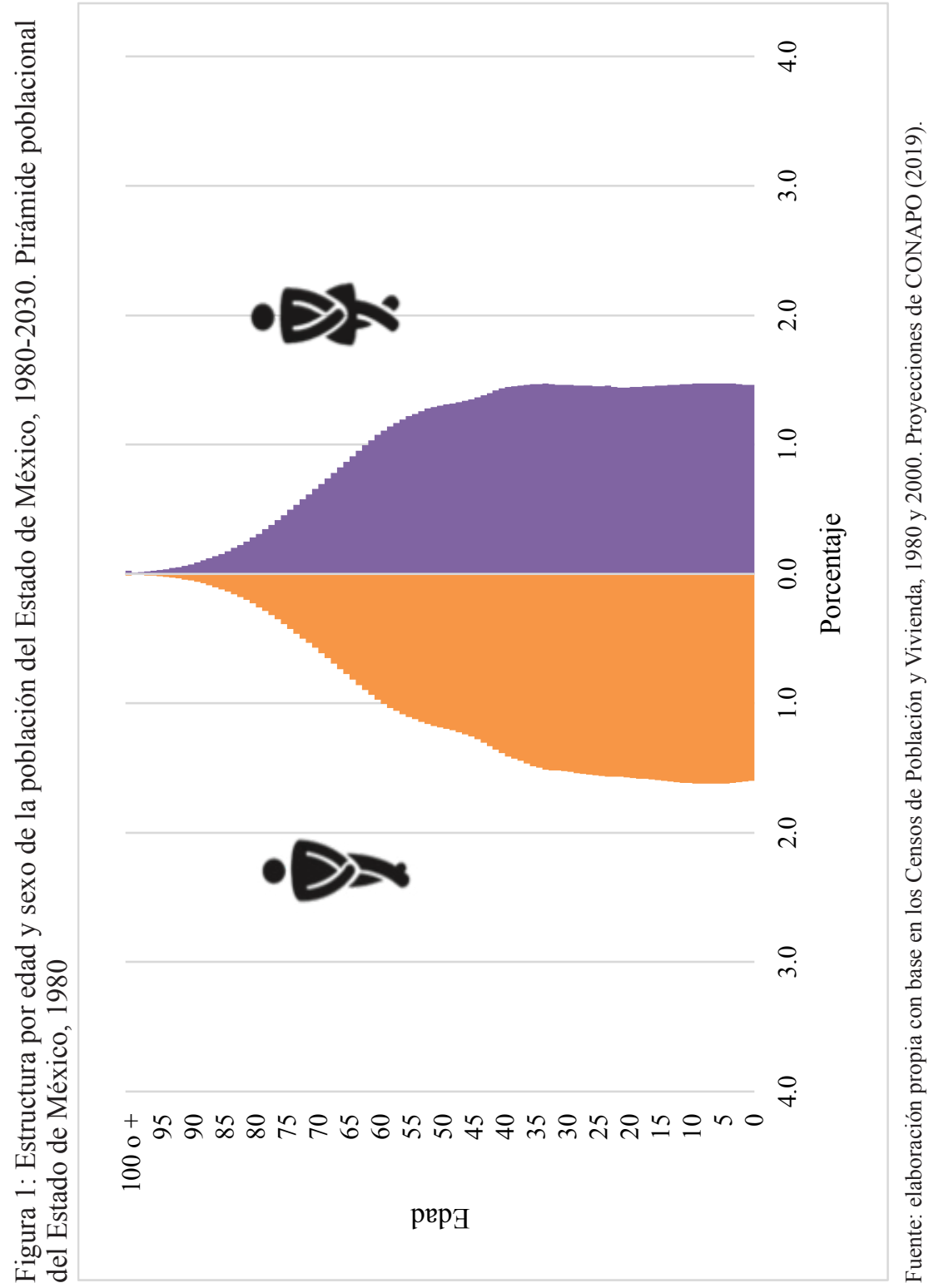


Mortalidad y proyecciones por causa de muerte en el Estado de México, 1980-2050 / Y.G. RÓMAN SÁNCHEZ et al.

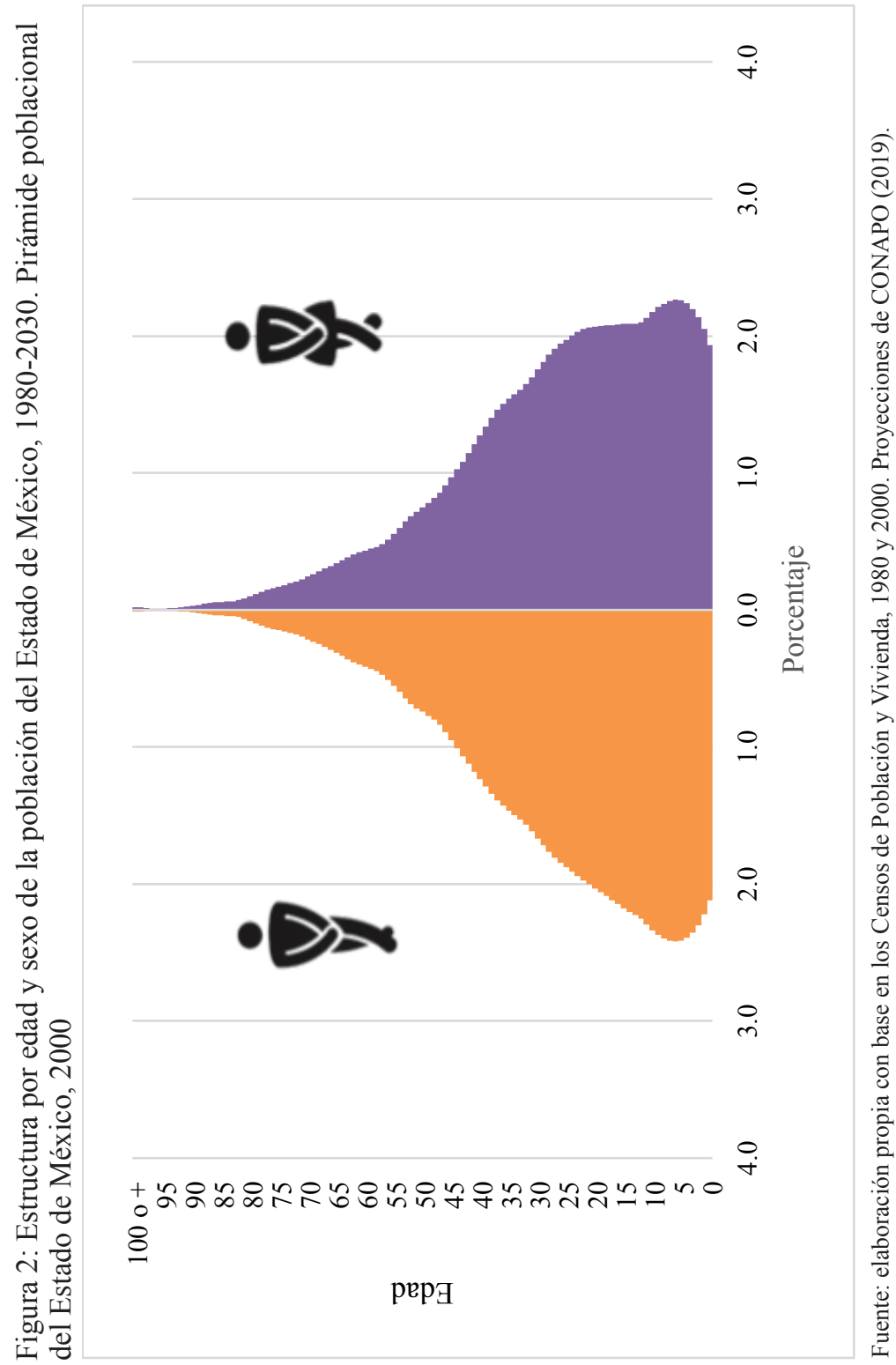




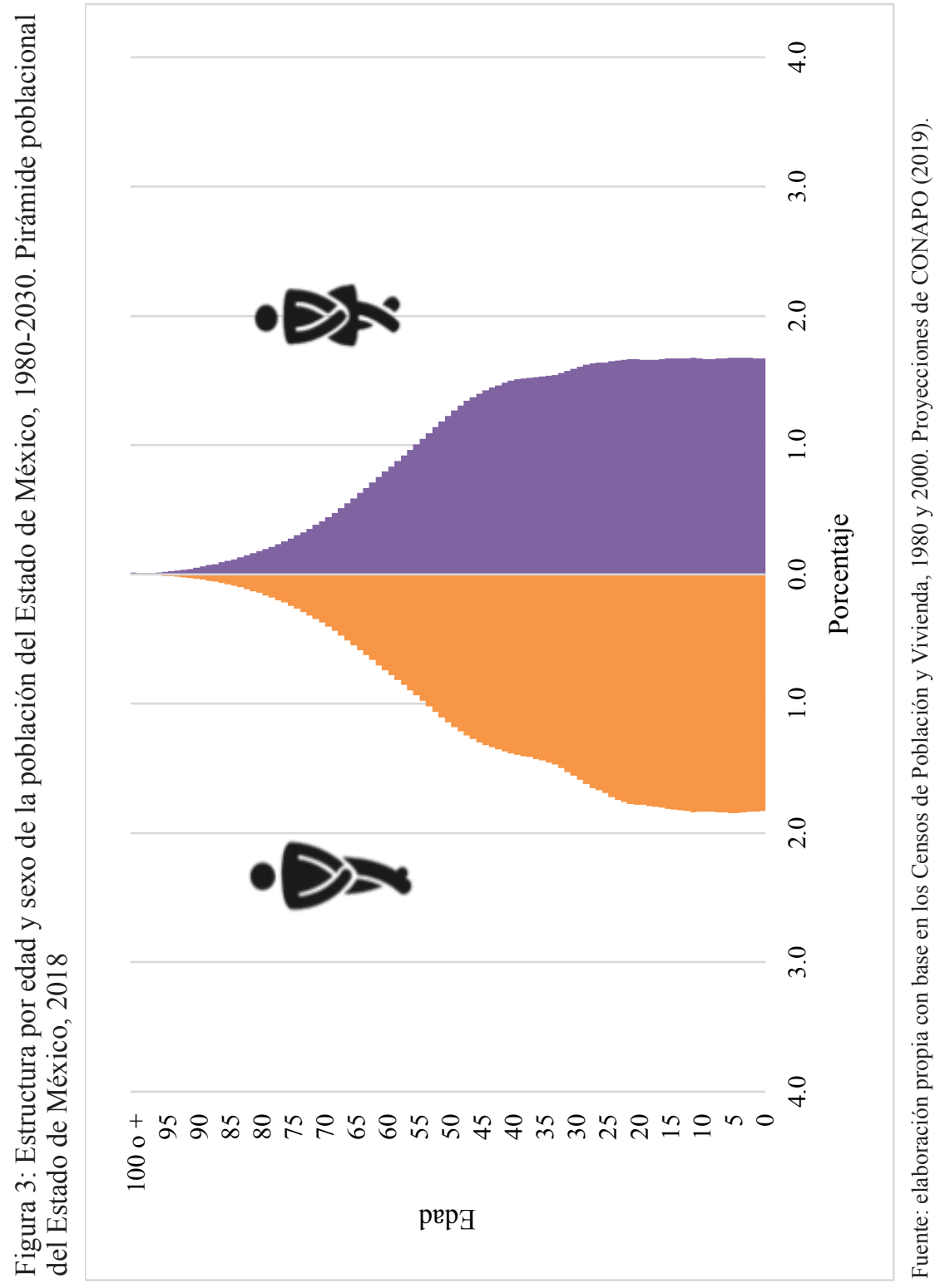


Mortalidad y proyecciones por causa de muerte en el Estado de México, 1980-2050 / Y.G. RÓMAN SÁNCHEZ et al.

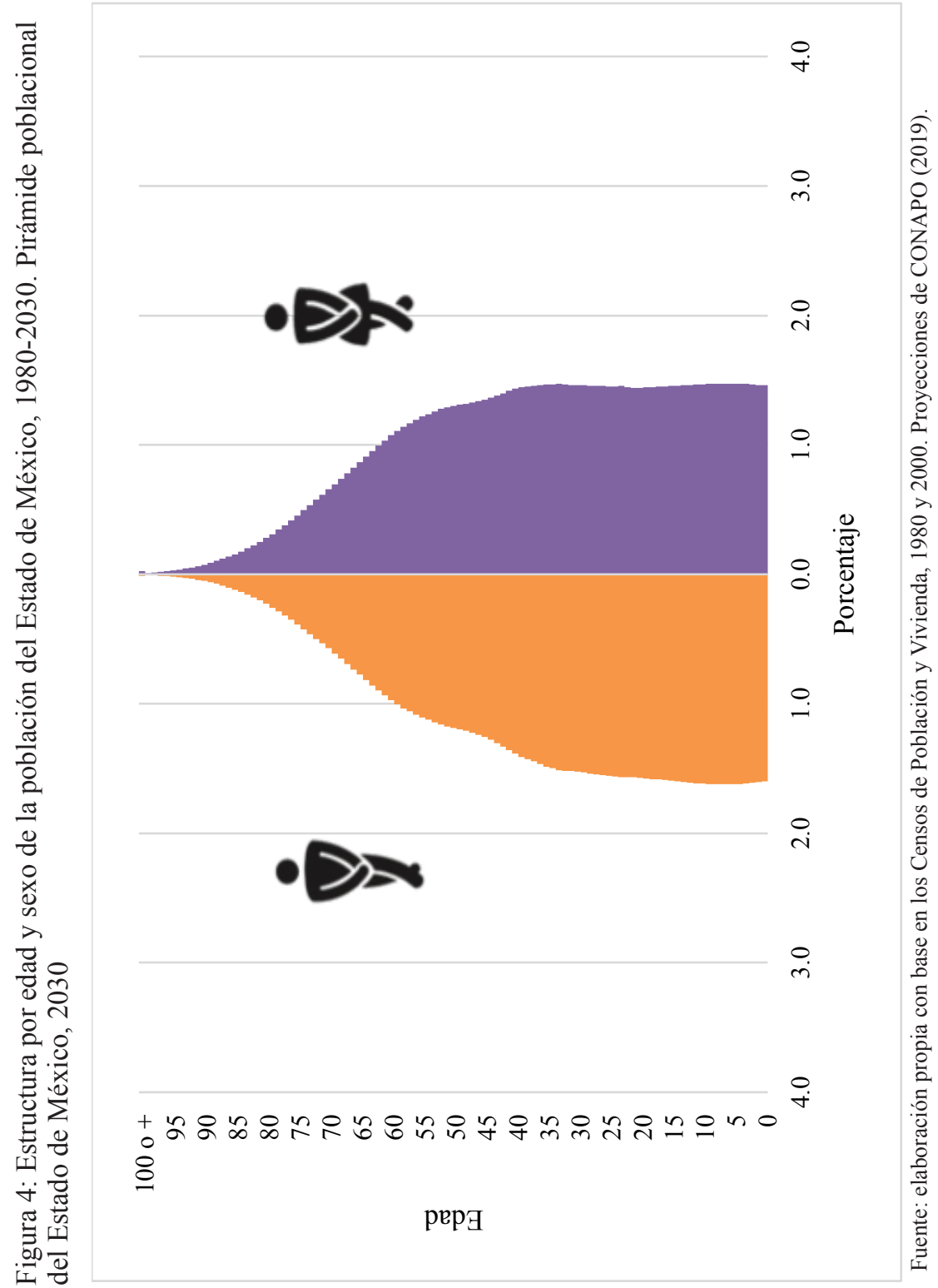


Tabla 1: Estado de México. Tasas de mortalidad según grupo de causas (defunciones por cada diez mil habitantes)

\begin{tabular}{|c|c|c|c|c|c|c|c|}
\hline \multirow[t]{2}{*}{ Grupo de causa } & \multicolumn{7}{|c|}{ Año } \\
\hline & 1980 & 1990 & 1995 & 2000 & 2005 & 2010 & 2015 \\
\hline \multicolumn{8}{|l|}{ Total } \\
\hline Ciertas enf. infecciosas y parasitarias (A00-B99) & 5.78 & 3.82 & 1.60 & 1.26 & 0.92 & 0.73 & 0.59 \\
\hline Tumores (neoplasias) (C00-D48) & 3.25 & 3.53 & 3.35 & 3.29 & 3.41 & 3.32 & 3.36 \\
\hline $\begin{array}{l}\text { Enf. endócrinas, nutricionales y metabólicas } \\
\text { (E00-E90) }\end{array}$ & 3.60 & 4.78 & 4.91 & 4.96 & 5.58 & 6.00 & 5.78 \\
\hline Enfermedades del sistema circulatorio (I00-I99) & 10.50 & 7.21 & 7.29 & 6.29 & 5.92 & 6.32 & 6.74 \\
\hline Enfermedades del sistema respiratorio (J00-J99) & 10.73 & 6.61 & 5.09 & 3.72 & 3.37 & 2.94 & 2.43 \\
\hline Enfermedades del sistema digestivo (K00-K93) & 7.47 & 5.61 & 5.02 & 4.18 & 3.74 & 3.34 & 3.21 \\
\hline Causas externas de movilidad y de mortalidad* & 12.01 & 7.77 & 5.06 & 3.91 & 3.63 & 3.91 & 4.22 \\
\hline \multicolumn{8}{|l|}{ Hombres } \\
\hline Ciertas enf. infecciosas y parasitarias (A00-B99) & 6.41 & 4.27 & 1.75 & 1.45 & 1.08 & 0.85 & 0.70 \\
\hline Tumores (neoplasias) (C00-D48) & 2.73 & 3.18 & 2.93 & 3.05 & 3.12 & 3.13 & 3.29 \\
\hline $\begin{array}{l}\text { Enf. endócrinas, nutricionales y metabólicas } \\
\text { (E00-E90) }\end{array}$ & 3.43 & 4.46 & 4.77 & 4.68 & 5.39 & 6.06 & 5.92 \\
\hline Enfermedades del sistema circulatorio (I00-I99) & 10.46 & 6.73 & 6.63 & 5.90 & 5.75 & 6.30 & 6.71 \\
\hline Enfermedades del sistema respiratorio (J00-J99) & 11.79 & 7.35 & 5.66 & 4.04 & 3.54 & 3.15 & 2.60 \\
\hline Enfermedades del sistema digestivo (K00-K93) & 10.14 & 7.98 & 7.02 & 5.85 & 5.21 & 4.59 & 4.27 \\
\hline Causas externas de movilidad y de mortalidad* & 19.29 & 12.61 & 8.20 & 6.39 & 5.79 & 6.35 & 6.77 \\
\hline \multicolumn{8}{|l|}{ Mujeres } \\
\hline Ciertas enf. infecciosas y parasitarias (A00-B99) & 5.19 & 3.39 & 1.45 & 1.07 & 0.78 & 0.62 & 0.48 \\
\hline Tumores (neoplasias) (C00-D48) & 3.77 & 3.89 & 3.75 & 3.52 & 3.68 & 3.49 & 3.41 \\
\hline $\begin{array}{l}\text { Enf. endócrinas, nutricionales y metabólicas } \\
\text { (E00-E90) }\end{array}$ & 3.76 & 5.08 & 5.03 & 5.22 & 5.77 & 5.94 & 5.65 \\
\hline Enfermedades del sistema circulatorio (I00-I99) & 10.49 & 7.67 & 7.91 & 6.67 & 6.08 & 6.36 & 6.79 \\
\hline Enfermedades del sistema respiratorio (J00-J99) & 9.70 & 5.91 & 4.54 & 3.42 & 3.21 & 2.73 & 2.26 \\
\hline Enfermedades del sistema digestivo (K00-K93) & 4.76 & 3.29 & 3.06 & 2.58 & 2.34 & 2.17 & 2.23 \\
\hline Causas externas de movilidad y de mortalidad* & 4.65 & 3.04 & 1.98 & 1.54 & 1.58 & 1.57 & 1.79 \\
\hline
\end{tabular}

* Para 1990 y 1995, las "Causas externas de movilidad y de mortalidad" se encontraban con el nombre de "Clasificación suplementaria de causas externas de traumatismo y envenenamiento".

Fuente: elaboración propia con base en Estadísticas vitales (1979, 1980, 1981, 1989, 1990, 1991, 1994, 1995,1996, 1999, 2000, 2001, 2004, 2005, 2006, 2009, 2010, 2011, 2014, 2015, 2016).

Por un lado, el aumento de las enfermedades endócrinas permite vislumbrar que poco a poco se han convertido en una preocupación de salud pública debido a la frecuencia con la que causan muertes en todos los grupos de edades, al pasar de cuatro muertes por cada diez mil personas en 
1980 a seis muertes por cada diez mil personas en 2015. Por otro lado, las defunciones por enfermedades del sistema circulatorio muestran un declive importante de 1980 a 1990, y una estabilidad entre 1990 y 2015.

Respecto a los hombres, las causas externas han sido de gran relevancia para este grupo de población, pues esta causa de muerte ha presentado una disminución importante para 2015 con respecto a 1980 pasando de 19 muertes a sólo siete por cada diez mil hombres. Las muertes a causa de enfermedades endócrinas han presentado un incremento de 50 por ciento en su frecuencia y las tasas de mortalidad por tumores se han mantenido casi estáticas de 1980 a 2015. Las enfermedades circulatorias presentaron un ligero aumento de seis a siete muertes por cada diez mil hombres de 2005 a 2015. En tanto, que el resto de las enfermedades presenta una tendencia a la baja.

$\mathrm{Al}$ analizar los grupos de causas de muerte por sexo, se observa que las enfermedades del sistema circulatorio han permanecido como la principal causa de muerte en mujeres durante el periodo de análisis. No obstante, es posible visualizar un declive importante pasando de aproximadamente 11 muertes a siete por cada diez mil mujeres. En contraste, los hombres presentan un decremento de alrededor de diez por ciento en las muertes por tumores durante el periodo analizado; mientras que las enfermedades del sistema digestivo, respiratorio, enfermedades infecciosas y causas externas han disminuido. Asimismo, las enfermedades del sistema respiratorio pasaron de causar diez muertes por cada diez mil mujeres en 1980 a dos por cada diez mil mujeres para 2015. Sin embargo, las muertes por enfermedades endócrinas en las mujeres, al igual que en los hombres, se presentan cada vez con mayor frecuencia, aunque para 2015 parece haber una disminución respecto a 2010.

En la Tabla 1, por un lado, se puede observar una tendencia hacia la baja de la mortalidad en general de 1980 a 2015, con un patrón donde prevalecen las enfermedades no transmisibles, es decir, gradualmente aumentan las enfermedades crónico-degenerativas. Por otro lado, se hace evidente que las enfermedades infecciosas y parasitarias no se han erradicado por completo. Esto sugiere un perfil epidemiológico mixto, el cual —en el contexto de la aparición del virus SARS-CoV-2 - ha generado nuevas incógnitas en el campo epidemiológico.

\section{Metodología PARA las PROYeCCIONES POR CAUSAS DE MUERTE}

El modelo general de mortalidad supone que la mortalidad de la población humana es una función positiva creciente de la edad. La supervivencia cae 
a cero a medida que aumenta la edad porque la mortalidad acumulada está creciendo (Tuljapurkar y Ryan, 2015), es decir, la probabilidad de muerte incrementa conforme se avanza en el proceso de envejecimiento.

A lo largo de la historia de la humanidad se han utilizado diversas funciones matemáticas para estimar el comportamiento de la mortalidad por edad; la primera propuesta formal fue en 1725 con el nombre La ley de DeMoivre, su inventor supuso que los sobrevivientes de la tabla de mortalidad decrecían linealmente a lo largo del rango de edades (Pitacco, 2003, Partida, s/f). Se encuentra también la función propuesta de Charles Babbage en 1823, donde se plantea que la función de supervivencia se comporta como una función cuadrática de la edad, en lugar de presentar un comportamiento lineal (Partida, s/f).

Posteriormente, en la nueva era de la ciencia actuarial, se presentó la propuesta de Benjamín Gompertz, pionero de un nuevo enfoque al modelado de supervivencia en 1825 (Pitacco, 2003; Partida s/f). La ley de Gompertz constituye una de las propuestas de modelado de supervivencia (Pitacco, 2003) más influyentes, puesto que constituye el modelo más célebre de mortalidad por edad adulta, donde la fuerza de la mortalidad aumenta exponencialmente con la edad (Tuljapurkar y Ryan, 2015). En la actualidad se ha podido probar empíricamente que la Ley de Gompertz es adecuada entre las edades que van de los 35 ó 45 a 85 años (Partida, s/f).

Debido a que la ley Gompertz no modela adecuadamente la mortalidad en edades avanzadas, han surgido nuevas propuestas. Tal es el caso de la investigación de Vaupel y Yashin (1985), Thatcher et al. (1998) y colaboradores, ellos sugieren que un modelo logístico con una asintótica describe la mortalidad en la vejez con mayor precisión puesto que la función logística también puede verse como resultado de un modelo en el que la mortalidad de Gompertz se modifica por una fragilidad multiplicativa (Tuljapurkar y Ryan, 2015).

Una vez conocida la Ley de Gompertz, ya no bastaba modelar el comportamiento de la mortalidad general, sino que cobró mayor relevancia el modelado de la mortalidad por causa de muerte. En este contexto, surge la Ley de Makeham en 1867, la cual muestra que todos los niveles de mortalidad podían ser representados mejor si se agregaba una constante a la Ley de Gompertz para tomar en cuenta las causas de muerte que no dependen de la edad. Por ejemplo: los accidentes o epidemias. Bajo esta ley se puede observar la (decreciente) mortalidad a edades muy jóvenes, así como la joroba de la mortalidad en edades de adultos jóvenes, y por último la mortalidad en edad adultas y ancianas (Pitacco 2003; Partida, s/f). 
Posteriormente Heligman y Pollard en 1980, siguiendo la lógica de Makeham, proponen tres curvas parametrizadas: una para la infancia, otra para las edades adultas y la tercera para la senectud, con el interés de ajustar satisfactoriamente el rango completo de la tasa instantánea de mortalidad (Pitacco, 2003; Partida, s/f.). Naciones Unidas (1988) realizó un ajuste de las funciones de Heligman y Pollard que es fácilmente implementable en computadora. La nueva ciencia actuarial inició el estudio del modelado de la mortalidad con la función Gompertz. No obstante, en el quehacer demográfico y actuarial se han ido desarrollando nuevas formas más precisas de modelado de la mortalidad. Tal es el caso de la Ley Makeham, los ajustes de Heligman y Pollard y la inclusión de la función Gamma para modelar mortalidad por diferentes causas de muerte.

La distribución gamma ha sido utilizada para las proyecciones de la mortalidad. Específicamente, para modelar la variable de fragilidad de la mortalidad (Vaupel et al., 1979; Wang y Brown, 1998) pues tiene como ventaja su manejabilidad y flexibilidad matemática (Wang y Brown, 1998). Esta distribución tiene sus bases en las primeras leyes de la mortalidad propuestas por Perks en 1932 (Wang y Brown,1998). Por ejemplo: en la investigación de Tuljapurkar y Ryan (2015) se usó la distribución gamma para presentar resultados analíticos de cómo la varianza en la edad a la muerte de un adulto depende de los parámetros de los modelos de mortalidad. Para ello usan la función de Gompertz con una logística, luego un modelo general de fragilidad multiplicativa con casos especiales de fragilidad gamma, y finalmente utilizan una función Gompertz con fragilidad gamma (Tuljapurkar y Ryan 2015). Esto último con la finalidad de conocer el comportamiento de la mortalidad general y modelar conjuntamente fragilidad a la muerte diferenciando por grupo de edad.

Antes de implementar el modelado de mortalidad por causa de muerte utilizando la función Gamma, se estandarizaron las tasas específicas de mortalidad por el método directo. Dicho procedimiento permite eliminar el efecto de la estructura por edades de la población (Primante y García, 1997); se utilizaron las tasas de mortalidad por edades quinquenales promedio de 1980, 1990, 1995, 2000, 2005, 2010 y 2015 y la estructura por edades de la población del año 2000 fue considerada población tipo. Para posteriormente aplicar el procedimiento de Primante y García (1997). Específicamente, el procedimiento para estimar los parámetros de la función gamma fue el siguiente:

Se determinaron las matrices:

$t=$ representa el $i$-ésimo año de la tasa estandarizada 
TEM $=$ tasa estandarizada en el $i$-ésimo año

$$
X=\left[\begin{array}{ccc}
1 & t_{1} & \operatorname{Ln}\left(t_{1}\right) \\
\vdots & \vdots & \vdots \\
1 & t_{n} & \operatorname{Ln}\left(t_{n}\right)
\end{array}\right] \quad Y=\left[\begin{array}{c}
\operatorname{Ln}\left(T E M_{1}\right) \\
\vdots \\
\operatorname{Ln}\left(T E M_{n}\right)
\end{array}\right] \quad X^{T}=\left[\begin{array}{ccc}
1 & \ldots & 1 \\
t_{1} & \ldots & t_{n} \\
\operatorname{Ln}\left(t_{1}\right) & \ldots & \operatorname{Ln}\left(t_{n}\right)
\end{array}\right]
$$

Posteriormente se estima la multiplicación de las matrices $X^{*} X^{\mathrm{T}}$, es decir:

$$
\begin{gathered}
W=X * X^{T}=\left[\begin{array}{ccc}
1 & t_{1} & \operatorname{Ln}\left(t_{1}\right) \\
\vdots & \vdots & \vdots \\
1 & t_{n} & \operatorname{Ln}\left(t_{n}\right)
\end{array}\right] *\left[\begin{array}{ccc}
1 & \ldots & 1 \\
t_{1} & \ldots & t_{n} \\
\operatorname{Ln}\left(t_{1}\right) & \ldots & \operatorname{Ln}\left(t_{n}\right)
\end{array}\right] \\
W=\left[\begin{array}{ccc}
1 * 1+t_{1} * t_{1}+\operatorname{Ln}\left(t_{1}\right) * \operatorname{Ln}\left(t_{1}\right) & \ldots & 1 * 1+t_{1} * t_{n}+\operatorname{Ln}\left(t_{1}\right) * \operatorname{Ln}\left(t_{n}\right) \\
\vdots & \vdots & \vdots \\
1 * 1+t_{n} * t_{1}+\operatorname{Ln}\left(t_{n}\right) * \operatorname{Ln}\left(t_{1}\right) & \ldots & 1 * 1+t_{n} * t_{n}+\operatorname{Ln}\left(t_{n}\right) * \operatorname{Ln}\left(t_{n}\right)
\end{array}\right]
\end{gathered}
$$

Asimismo, se estima la matriz inversa de la multiplicación de matrices que se obtuvo en el paso anterior.

$$
M=\left[W^{-1}\right]=\frac{\operatorname{Adj}(W)}{|W|}
$$

Para casi finalizar se multiplica la matriz inversa $M$ con la matriz traspuesta de $X^{\mathrm{T}}$

Finalmente, los parámetros de la función gamma se obtienen de la multiplicación de la matriz anterior con la matriz $Y$.

$$
A=\left[\begin{array}{l}
a \\
b \\
c
\end{array}\right]
$$

Para obtener las nuevas tasas los parámetros se sustituyen en:

$$
Y(x)=e^{a+b x+c * \operatorname{Ln}(x)}
$$

La validación interna del modelo se llevó a cabo al estimar las tasas estandarizadas para los periodos anteriores y se observó que el error fue 
muy bajo, menor de medio punto porcentual. Asimismo, cabe destacar que la calidad de los datos, mala declaración de la edad, la información de los Censos de Población y Vivienda se corrigieron usando el método de los multiplicadores de Sprague, mientras que la información de las defunciones se suavizó usando el promedio de éstas.

\section{Proyecciones de la mortalidad Según causa de muerte Para la POBLACIÓN MEXIQUENSE, 2020-2050}

Utilizando las proyecciones de la mortalidad del CONAPO (2019) se desarrolló la Figura 5, en la cual se puede observar la estructura por edad y sexo de las defunciones de la población mexiquense para el año 2020 y 2030.

También se puede resaltar que, en 2030 la mortalidad sufrirá un descenso de la población de 0 años hasta las personas menores de 60 años respecto a la esperada en 2020. A partir de los 60 años, el porcentaje de mortalidad presenta un aumento bastante notorio de diez puntos porcentuales, al pasar de 63.2 por ciento de 2020 a 73.9 por ciento en 2030; donde las mujeres son las principales afectadas de este aumento en ambos años, sobre todo en edades más avanzadas aproximadamente desde los 70 años o más (CONAPO, 2019).

A partir de las proyecciones estimadas se espera que para la población total las enfermedades infecciosas, del sistema respiratorio, sistema digestivo, tumores y causas externas se diluyen a lo largo de los años como causas de mortalidad importantes, con tres o menos muertes por cada diez mil personas. Mientras que las muertes por enfermedades del sistema circulatorio y las enfermedades endócrinas ascienden con fuerza desde 2020 hasta 2050. En el caso de las enfermedades del sistema circulatorio se presenta un aumento de 8 a 19 muertes por cada diez mil personas, en promedio, es decir, se incrementan 2.5 veces las muertes en el periodo de estudio. Por otro lado, las enfermedades endócrinas ascienden a un ritmo equiparable de 2.4 veces, pues pasaran de causar siete muertes por cada diez mil personas en 2020 a 17 defunciones en 2050 por cada diez mil personas (Figura 6).

Para el sexo masculino se puede apreciar un crecimiento importante en las enfermedades del sistema circulatorio y endócrinas, pues pasan de generar ocho a 23 muertes entre cada diez mil hombres, y de siete a 25 muertes por cada diez mil hombres, respectivamente. Es decir, se visualiza de un crecimiento en 2050 , mayor o igual a tres veces al presentado en 2020 (Figura 7). 


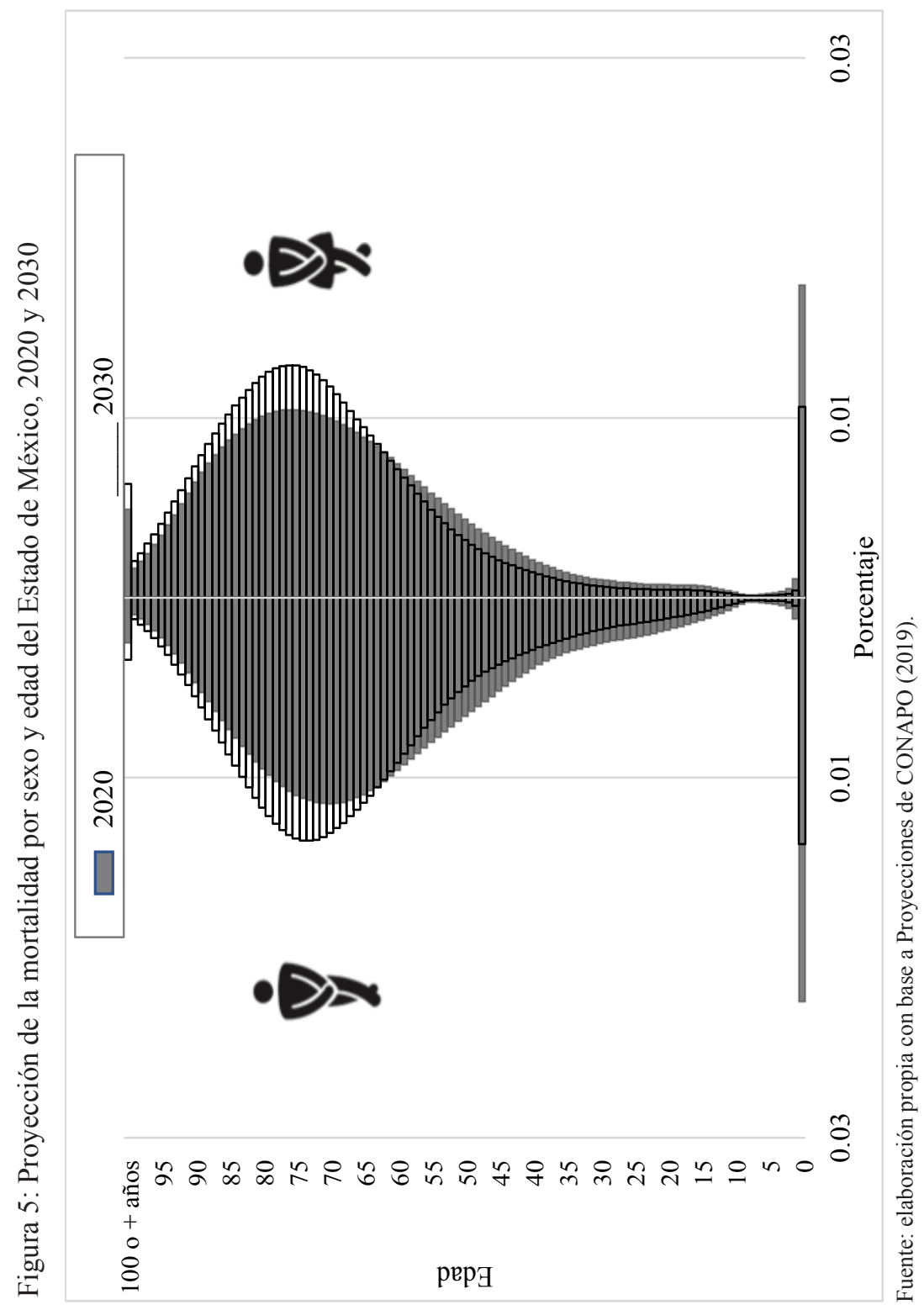


Mortalidad y proyecciones por causa de muerte en el Estado de México, 1980-2050 / Y.G. RÓMAN SÁNCHEZ et al.
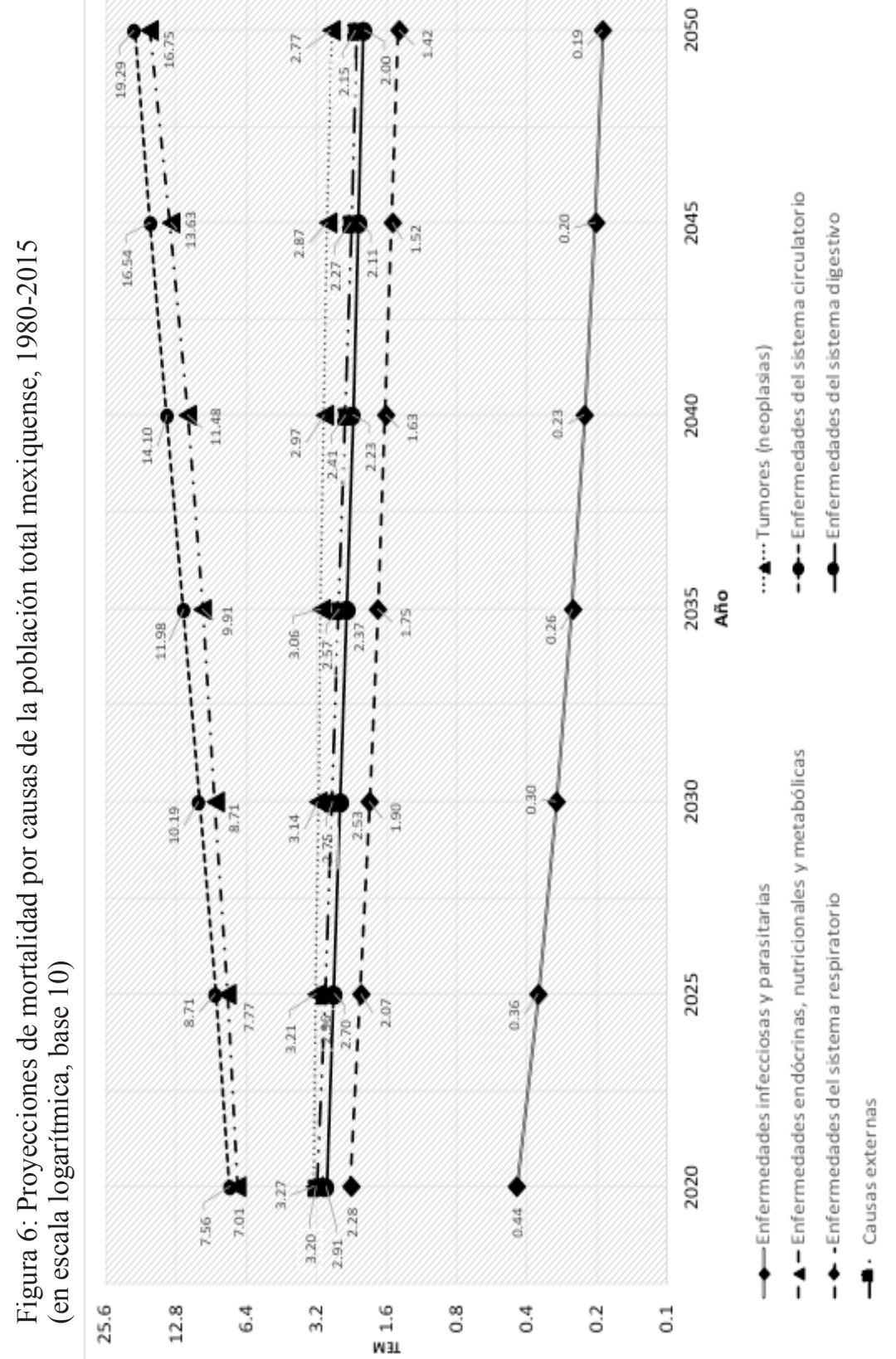

กิ

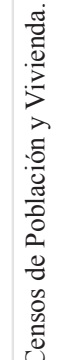

啇

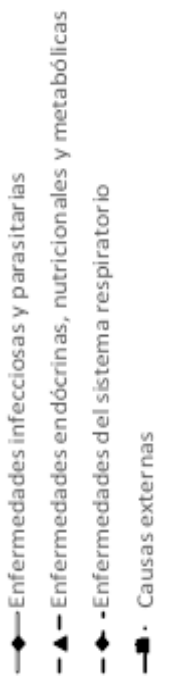

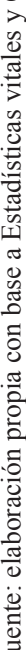


Figura 7: Proyecciones de mortalidad por causas de la población masculina total mexiquense, 1980-2015 (en escala logarítmica, base 10)

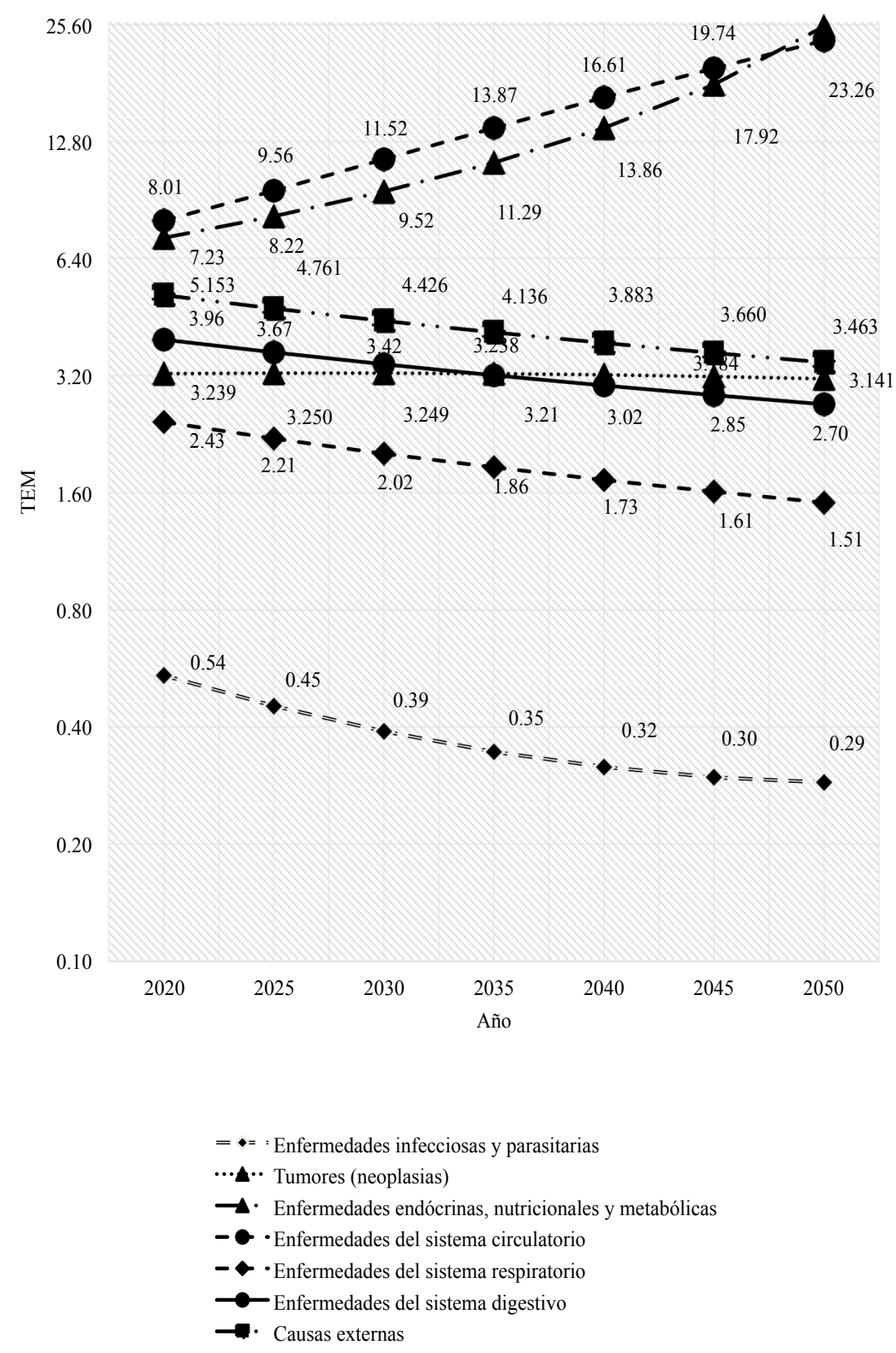

Fuente: elaboración propia con base a Estadísticas vitales y Censos de Población y Vivienda. 
Figura 8: Proyecciones de mortalidad por causas de la población femenina total mexiquense, 1980-2015 (escala logarítmica, base 10)

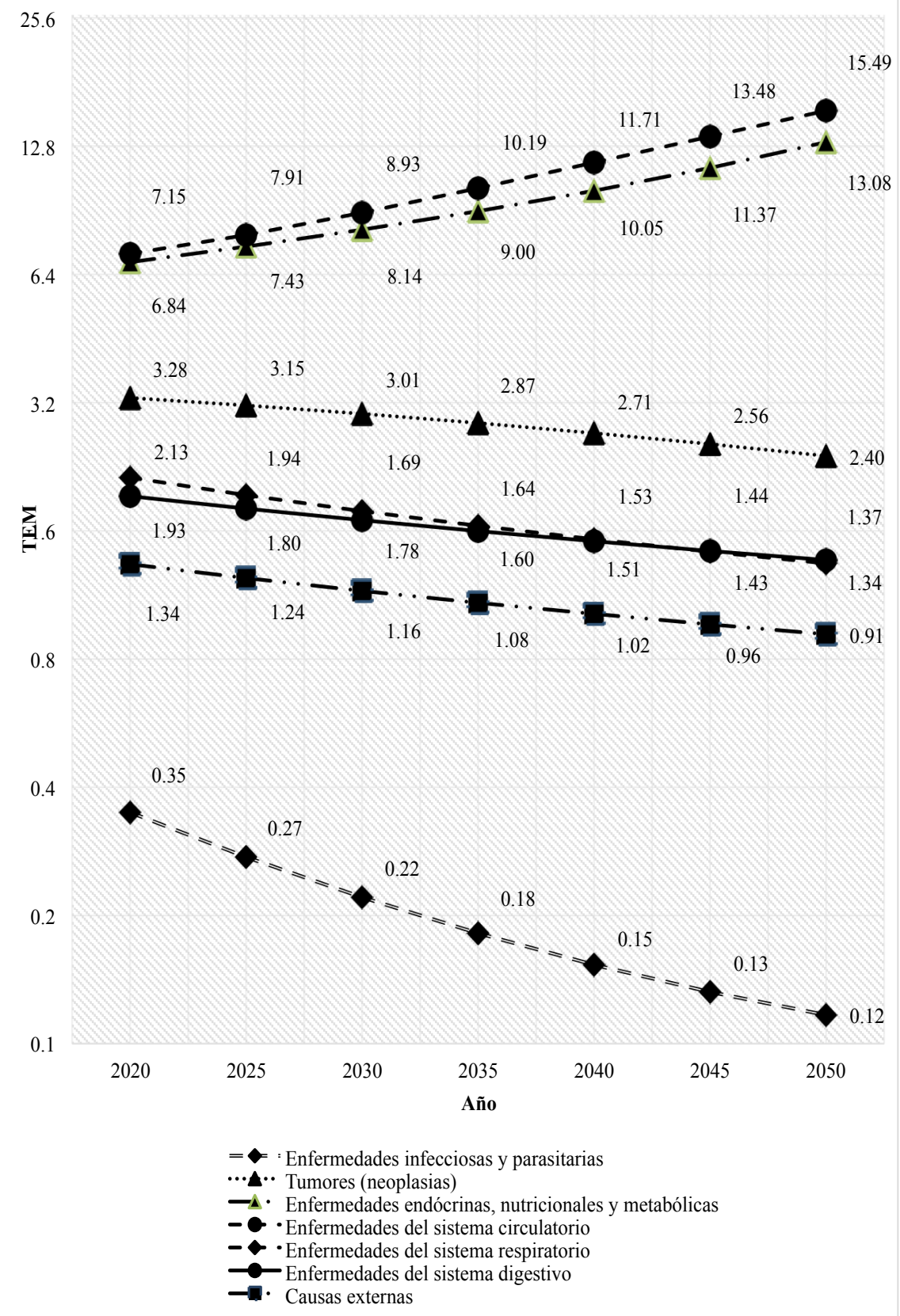

Fuente: elaboración propia con base a Estadísticas vitales y Censos de Población y Vivienda. 
Otro punto por resaltar es que las muertes causadas por tumores encuentran un equilibrio que se mantiene alrededor de las tres muertes entre cada diez mil hombres durante el periodo analizado. Las enfermedades restantes sufren, por su parte, un decremento casi constante. En el caso de las causas externas se reduce de cinco a poco menos de cuatro muertes por cada diez mil hombres; las enfermedades infecciosas causan una muerte por cada diez mil hombres; las enfermedades del sistema respiratorio pasan de dos a una muerte por cada diez mil varones, todo en los 30 años proyectados (Figura 7).

$\mathrm{Al}$ igual que los varones, las mujeres presentan incrementos importantes en las muertes por enfermedades del sistema circulatorio y enfermedades endócrinas. Sin embargo, los aumentos son menos bruscos. En la Figura 8 se muestra las proyecciones de mortalidad por causa en la población femenina, donde la primera causa - del sistema circulatorio- para 2050 existirá el doble de muertes que se presentarán en 2020; mientras que para el segundo padecimiento enfermedades endócrinas se proyecta que para 2020 será causante de siete muertes por cada diez mil mujeres y para 2050 de 13 decesos por cada diez mil mujeres. El resto de las enfermedades experimentarán un deceso por cada diez mil mujeres, de aproximadamente una muerte menos en el periodo de 30 años, con tasas de descenso casi constante.

Como fue posible observar, las proyecciones de la mortalidad estiman el aumento de las defunciones por enfermedades crónico-degenerativas. Situación que traerá aparejados una serie de retos; constituyendo el reto más acuciante el incremento en la cantidad de demanda de atención y servicios médicos, tanto por el aumento de adultos mayores que incrementarán su esperanza de vida, como por las necesidades de atención y costo de estos padecimientos (Palloni, Pinto y Peláez, 2002). Aunado a ello, es importante considerar las implicaciones del incremento del tiempo de exposición al que estarán expuestas las personas que vivan con estas enfermedades, debido a que las enfermedades crónico-degenerativas se exacerban con la edad y su atención en salud y cuidado implican un alto costo para el individuo, las familias y el Estado.

\section{Discusión de los resultados}

Los resultados de las proyecciones dejan entrever los problemas económicos y financieros que se presentarán con el comportamiento futuro de la mortalidad, debido al elevado costo que generarán las enfermedades crónico-degenerativas en la población mexiquense. Al respecto, la Organización 
Mundial de la Salud (2011) pronostica que las enfermedades crónicas no transmisibles en adultos mayores de los países en desarrollo (enfermedad cardíaca, el cáncer y la diabetes) generarán pérdidas económicas: se estima un monto de 83 mil millones de dólares entre 2006 y 2015 en estos países (World Health Organization, 2011).

En particular en México, los costos por diabetes e hipertensión en tres diferentes instancias de salud son muy altos. Por destacar un ejemplo, en el Instituto Mexicano del Seguro Social (IMSS) el costo por diabetes fue de 46 millones de 2001 a 2003 y 55 millones de 2004 a 2006. Mientras que en la Secretaría de Salud fue de 16 millones y 24 millones para los mismos periodos. En el Instituto de Seguridad y Servicios Sociales de los Trabajadores del Estado (ISSSTE) entre 2004 y 2006 los costos fueron de 10 y 11 millones, respectivamente (Arredondo, 2001; Villalobos y Ham, 2012).

El estudio de Ávila-Burgos, et. al; (2009) también abona información al respecto. Sólo en 2006 el gasto que provocaron enfermedades como la diabetes mellitus, obesidad y enfermedades cardiovasculares en todos los grupos de edad fue de 39 mil 911 millones de pesos, equivalente a siete por ciento del gasto total en salud pública y 0.4 por ciento del PIB registrado en dicho año. Información más específica indica que 55.5 por ciento del gasto fue en enfermedades cardiovasculares, 40.6 por ciento en diabetes mellitus y 14.1 por ciento en obesidad, se concentraron en personas de 60 años y más (Arredondo, 2001; Villalobos y Ham, 2012).

Con lo anterior, es posible mencionar que, con el aumento pronosticado de las defunciones por enfermedades crónico-degenerativas, el Estado de México tiene grandes retos que atender, no solo el aumento de los adultos mayores en la entidad, sino todas las demandas que implica ello: salud, infraestructura, servicios, y, por supuesto el incremento del gasto que conllevan estos padecimientos.

En este sentido, a partir de las proyecciones estimadas sobre la mortalidad por causas de la población mexiquense es posible notar que las enfermedades endocrinas, nutricionales y metabólicas, será el grupo que podría generar los costos más elevados tanto en el ámbito público y privado, valdría la pena realizar investigaciones al respecto. Ahora bien, estos costos repercutirán tanto en la distribución del gasto privado (hogares) como público (instituciones de salud públicas), de ahí que resulta urgente implementar alguna política pública a través de programas sociales de atención en alimentación, salud y cuidados de la población mexiquense de manera pronta para ver resultados a mediano y largo plazo. Además, del incremento económico destaca el aumento en las horas para el cuidado de 
adultos mayores, elevados grados de dependencia funcional y limitaciones físicas, lo que conducirá a cambios de roles en la sociedad.

\section{Conclusiones}

A partir de los resultados obtenidos es posible destacar cinco aspectos a considerar en materia de política pública y poblacional. Primero, el cambio de las estructuras por edad de la población en proceso de envejecimiento demográfico en el Estado de México continuará en las siguientes décadas, se espera que se registre un total de 2.97 millones de adultos mayores en 2030. En este sentido es relevante aplicar políticas públicas encaminadas a proteger a este grupo, y en general a toda la población, con el objetivo de reducir la desigualdad, pobreza, discriminación, vulnerabilidad, entre otros factores, que se presenta en edades avanzadas; con el propósito de otorgar mejores condiciones de vida en esta etapa de la vida.

Segundo, el grupo de los adultos mayores es el que concentra el mayor porcentaje de defunciones. Cabe resaltar que la tasa de mortalidad en los últimos quince años oscila entre 29 y 30 defunciones (por mil). Lo anterior implica que los progresos para reducir la mortalidad son más lentos pero que mediante mecanismos sociales eficientes, mejoras en la calidad de vida de la población, programas de salud pública, importación médica, progreso económico, programas de integración y desarrollo social se puede disminuir la mortalidad en edades avanzadas por debajo del nivel actual.

Tercero, según la diferenciación por sexo se puede concluir que en 1980 predominaban las enfermedades circulatorias y las enfermedades respiratorias para hombres y mujeres; mientras que en tercer lugar se colocaban las defunciones ocasionadas por enfermedades del sistema digestivo para el caso de los hombres y las enfermedades endocrinas en la población femenina. Por otra parte, en 2015 los decesos para ambos sexos eran originados por enfermedades del sistema circulatorio, las enfermedades endócrinas y los tumores. Con base en lo anterior, es posible corroborar la existencia de un perfil epidemiológico mixto en el Estado de México.

Cuarto, a partir de las proyecciones de la mortalidad es posible pronosticar que las principales causas de muerte en 2050 serán aquellas asociadas a enfermedades crónico-degenerativas. Lo cual deja entrever el incremento de gastos económicos en salud, atención médica y farmacéutica, cuidado de adultos mayores, elevados grados de dependencia funcional y limitaciones físicas, lo que conducirá a cambios de roles en la sociedad.

Finalmente, quinto, se recomienda a los tomadores de decisiones implementar políticas de salud enfocadas a la prevención con el objetivo de 
erradicar enfermedades infecciosas para evitar un resurgimiento de éstas en un futuro y retomar mejores estrategias que ayuden a prevenir y controlar las enfermedades crónico-degenerativas. Esto último para evitar o disminuir el elevado impacto económico, sanitario y social que conlleva el gradual envejecimiento poblacional. Es decir, es preciso planear mecanismos que sean costo-efectivos a mediano y largo plazo, con el propósito de evitar colapsos en los sistemas de salud, así como no generar un déficit en las finanzas públicas.

Por último, agregar que la situación tan complicada por la que atraviesa el país y la entidad mexiquense, en cuanto al colapso de los hospitales por los infectados del virus SaRs-CoV-2 que necesitan intubación, se debe, en gran medida, a las comorbilidades (diabetes, hipertensión, obesidad) de una cantidad importante de mexicanos y mexiquenses; de ahí que resulte urgente implementar un programa continuo de atención prioritaria para el control y prevención de enfermedades crónico degenerativas como parte de la política pública para mejorar la salud.

\section{REFERENCIAS BIBLIOGRÁFICAS}

Agar, L., 2001, "Envejecimiento en América Latina y el Caribe: Hechos sociodemográficos y reflexiones éticas", en Acta Bioethica, 27-41.

Ávila-Burgos L, Cahuana-Hurtado L, González-Domínguez D, Aracena-Genao B, Montañez-Hernández JC, Serván-Mori EE, Rivera-Peña G., 2009, Cuentas en diabetes mellitus, enfermedades cardiovasculares y obesidad, México 2006. Ciudad de México/Cuernavaca, México: Instituto Nacional de Salud Pública.

INEGI, 1980, Censo de Población y Vivienda, 1980, INEGI, consultado el 4/11/2018. INEGI, 1990, Censo de Población y Vivienda, 1990, INEGI, consultado el 4/11/2018. INEGI, 2000, Censo de Población y Vivienda, 2000, INEGI, consultado el 4/11/2018. INEGI, 2010, Censo de Población y Vivienda, 2010, INEGI, consultado el 4/11/2018. CONAPO, 2019, Proyecciones de la Población 2010-2050: Datos de Proyecciones. Disponible en http://www.conapo.gob.mx/es/CONAPO/Proyecciones_Datos

INEGI, 1995, 2005, Conteo de Población y Vivienda, 1995, 2005, INEGI, consultado el 4/11/2018.

INEGI, 1979, 1980, 1981, 1989, 1990, 1991, 1999, 2000, 2001, 2009, 2010, 2011, Estadísticas vitales, INEGI, consultado el 4/11/2018.

Ham, R., 1999, Conceptos y significados del envejecimiento en las políticas de población. 
INEGI, 2020, Estadisticas vitales, defunciones generales. https://www.inegi. org.mx/sistemas/olap/consulta/general_ver4/MDXQueryDatos.asp?\#RegreSo\&c $=11144$

Naciones Unidas, 1988, MORTPAK - The United Nations Software Package for Mortality Measurement: Batch-oriented Software for the Mainframe Computer. ST/ESA/SER.R/78.

OMS, 2003, Informe sobre la salud en el mundo 2003: Forjemos el Futuro. (Cap. 6 Epidemias mundiales desatendidas: tres amenazas crecientes). Ginebra: Organización Mundial de la Salud (OMS). Recuperado el 11/01/2019 de http://www. who.int/whr/2003/en/Chapter6-es.pdf

Organización Panamericana de la Salud, 2001, Módulos de principios de epidemiología para el control de enfermedades.

Partida, Virgilio s/f., Notas para un curso de análisis demográfico. Facultad Latinoamericana de Ciencias Sociales. Sede Académica México.

Pitacco, Ermanno, 2003, Survival models in actuarial mathematics: from halley to longevity risk, Dipartimento di Matematica Applicata "B. de Finetti", University of Trieste, 7th International Congress Insurance: Mathematics \& Economics, ISFA, Lyon, June.

Primante, Domingo; García, Víctor, 1997, Apuntes de clase. XX Curso Regional Intensivo de Análisis Demográfico. Vol. 4, Mortalidad. Disponible en http://archivo.cepal.org/pdfs/1997/S9700584.pdf

Riley y Alter, 1990, The epidemiologic transition and morbidity. Working Paper núm 10 (Population Institute for Research and Training/Indiana University).

Romo, C., 2008, Envejecimiento Demográfico y Vejez del Sistema de Salud en México.

Rózga, R., 1994, "Desarrollo económico y población en el Estado de México”, en Papeles de Población, núm. 4-5, Universidad Autónoma del Estado de México, Toluca, México, pp. 113-128.

Sime, L., 2011, "Hacia una sociedad saludable: La educación ante la transición epidemiológica mundial", en Revista Electrónica Diálogos Educativos, 110-131.

Thatcher, AR.; Kannisto, V.; Vaupel, JW., 1998, Monographs on Population Aging. Odense University Press; Odense, Denmark, The force of mortality at ages 80 to $120 ;$ p. 5 .

Tuljapurkar, Shripad y Ryan, Edwards, 2015, "Variance in Death and Its Implications for Modeling and Forecasting Mortality", in Demogr Res. ; 24: 497-526, doi:10.4054/DemRes.2011.24.21

Vaupel, J.W. y Yashin, A.I., 1985, “Heterogeneity's Ruses: Some Surprising Effects of Selection on Population Dynamics", in Journal of the American Statistical Association 39, no. 3, pp: 176-185. 
Villa, M., y Rivadeneira, 2000, El proceso de envejecimiento de la población de América Latina y el Caribe: una expresión de la transición demográfica.

Villalobos A. y Ham R., 2012, “Costos de atención médica en la vejez”. Presentado en la XI Reunión de la Sociedad Mexicana de Demografía. Colegio de México.

Wang, Shaun y Brown, Robert, 1998, “A Frailty Model for Projection of Human Mortality Improvements", in Journal of Actuarial Practice 1993-2006, no. 95. University of Nebraska.

World Health Organization, 2011, Global Health and Aging. Washington: US National Institute on Aging, National Institutes of Health.

Vaupel, J.W., Manton, K.G. y Stallard, E., 1979, “The Impact of Heterogeneity in Individual Frailty on the Dynamics of Mortality", in Demography 16, no. 3, pp: 439-454.

Frenk, J., Frejka, T., \& Bobadilla, J., 1991, “La transición epidemiológica en América Latina", en Bol of Sanit Panam., 485-496.

Santos, M., 1996, "Envejecimiento demográfico. Diferencia por género", en Revista Española de Investigaciones Sociológicas, 177-190.

Vera, M., 1999, La teoría de la transición epidemiológica. México: El Colegio Mexiquense.

Bernabeu, J. y Robles, E., 2000, "Demografía y problemas de salud. Unas reflexiones críticas sobre los conceptos de transición demográfica y sanitaria", en UNED Política y Sociedad, 35, Dpt. de Sociología III (Tendencias sociales). Madrid, pp. 45-54.

Arredondo, A., 2001, "Requerimientos financieros para la demanda de servicios de salud por diabetes e hipertensión en México 2001-2003”, en Revista de Investigación Clínica, vol. 53, núm. 5, pp: 422-429.

Palloni A., Pinto G. \& Peláez M., 2002, "Demographic and health conditions of ageing in Latin America and the Caribbean", in International Journal of Epidemiology, vol. 31, núm. 4, pp. 762-771.

Whittembury, A., 2007, El perfil epidemiológico y el análisis de situación del país.

Cabrera, F., 2010, “Transición demográfica, transición epidemiológica: ¿Hacia dónde transita el Caribe?”, en Centro de Estudios Demográficos, 148-165.

Paz, J., 2010, Envejecimiento y empleo en América Latina y el Caribe. Suiza.

Pimienta, R.; Vera, M.; Tapia, J.; Orozco, M. E., 2015, "Evolución histórica de la población del Estado de México", en Quivera, vol. 17, núm. 2, Universidad Autónoma del Estado de México Toluca, México, pp. 109-138

Canales, A., 2015, "Inmigración y envejecimiento en Estados Unidos. Una relación por descubrir", en Estudios Demográficos y Urbanos, El Colegio de México.

INEGI, 2015, Encuesta Intercensal 2015, consultado el 4/11/2018. 


\section{RESUMEN CURRICULAR DE LOS AUTORES}

\section{Yuliana Gabriela Román Sánchez}

Doctora en Ciencias Económico-Administrativas por la UAEMéx. Maestra en Demografía por El Colegio de México. Licenciada en Actuaría Financiera por la UAEMéx. Profesora-Investigadora del Centro de Investigación y Estudios Avanzados de la Población (CIEAP) de la UAEMéx. Miembro del Sistema Nacional de Investigadores (SNI) Nivel I. Líneas de investigación: Desigualdad y Vulnerabilidad Demográfica: Temas: mercados de trabajo de la población joven, mortalidad, precariedad laboral y envejecimiento demográfico.

Dirección electrónica: ygromans@uaemex.mx; madon.d126@gmail.com

Registro ORCID: https://orcid.org/0000-0001-8571-9660

\section{Hugo Montes de Oca Vargas}

Economista por la Facultad de Economía de la UAEM. Maestro en Demografía por El Colegio de la Frontera Norte (COLEF), cuenta con estudios de Doctorado en Estudios de Población en El Colegio de México (COLMEX). Profesor-Investigador del Centro de Investigación y Estudios Avanzados de la Población (CIEAP) de la UAEM, con Perfil Deseable PROMEP. Docente en programas de licenciatura de la Facultad de Economía y de la Facultad de Enfermería y Obstetricia de la UAEM. Ha publicado artículos en revistas indizadas y es coautor de varios capítulos de libro. Áreas de interés: dinámica demográfica, envejecimiento demográfico, familia y envejecimiento, migración y envejecimiento, salud y envejecimiento y mercado de trabajo de los adultos mayores.

Dirección electrónica: huvic100@hotmail.com; hmontesdeocav@uaemex.mx

Registro ORCID: https://orcid.org/0000-0002-8844-7717

\section{José Antonio Soberón Mora}

Es maestro en demografía por El Colegio de la Frontera Norte (COLEF), Doctor en Urbanismo por la Universidad Autónoma del Estado de México (UAEMex), profesor investigador en el Centro de Investigación y Estudios Avanzados de la Población (CIEAP-UAEMex). Actualmente trabaja en los proyectos: Migración interna en México; Migración interna en la región centro de México.

Dirección electrónica: josesoberon2004@yahoo.com

Registro ORCID: https://orcid.org/0000-0003-2812-100x

Artículo recibido el 19 de junio de 2019 y aprobado el 25 de mayo de 2020. 